\section{Michigan Technological 1 в 8 5 University}

Michigan Technological University Digital Commons @ Michigan Tech

\title{
A comparison of hyperelastic constitutive models applicable to shear wave elastography (SWE) data in tissue-mimicking materials
}

\author{
David P. Rosen \\ Michigan Technological University \\ Jingfeng Jiang \\ Michigan Technological University
}

Follow this and additional works at: https://digitalcommons.mtu.edu/biomedical-fp

\section{Recommended Citation}

Rosen, D. P., \& Jiang, J. (2019). A comparison of hyperelastic constitutive models applicable to shear wave elastography (SWE) data in tissue-mimicking materials. Physics in Medicine \& Biology. http://dx.doi.org/10.1088/1361-6560/ab0137

Retrieved from: https://digitalcommons.mtu.edu/biomedical-fp/42 
ACCEPTED MANUSCRIPT

\section{A comparison of hyperelastic constitutive models applicable to shear wave elastography (SWE) data in tissue-mimicking materials}

To cite this article before publication: David P Rosen et al 2019 Phys. Med. Biol. in press https://doi.org/10.1088/1361-6560/ab0137

\section{Manuscript version: Accepted Manuscript}

Accepted Manuscript is "the version of the article accepted for publication including all changes made as a result of the peer review process, and which may also include the addition to the article by IOP Publishing of a header, an article ID, a cover sheet and/or an 'Accepted

Manuscript' watermark, but excluding any other editing, typesetting or other changes made by IOP Publishing and/or its licensors"

This Accepted Manuscript is (c) 2018 Institute of Physics and Engineering in Medicine.

During the embargo period (the 12 month period from the publication of the Version of Record of this article), the Accepted Manuscript is fully protected by copyright and cannot be reused or reposted elsewhere.

As the Version of Record of this article is going to be / has been published on a subscription basis, this Accepted Manuscript is available for reuse under a CC BY-NC-ND 3.0 licence after the 12 month embargo period.

After the embargo period, everyone is permitted to use copy and redistribute this article for non-commercial purposes only, provided that they adhere to all the terms of the licence https://creativecommons.org/licences/by-nc-nd/3.0

Although reasonable endeavours have been taken to obtain all necessary permissions from third parties to include their copyrighted content within this article, their full citation and copyright line may not be present in this Accepted Manuscript version. Before using any content from this article, please refer to the Version of Record on IOPscience once published for full citation and copyright details, as permissions will likely be required. All third party content is fully copyright protected, unless specifically stated otherwise in the figure caption in the Version of Record.

View the article online for updates and enhancements. 


\title{
A Comparison of Hyperelastic Constitutive Models Applicable to Shear Wave Elastography (SWE) Data in Tissue-Mimicking Materials
}

\author{
D P Rosen ${ }^{1}$, J Jiang ${ }^{1,2}$ \\ ${ }^{1}$ Department of Biomedical Engineering, Michigan Technological University, \\ Houghton, Michigan, USA \\ ${ }^{2}$ Department of Mechanical Engineering and Engineering Mechanics, Michigan \\ Technological University, Houghton, Michigan, USA
}

\begin{abstract}
Shear wave elastography (SWE) techniques have received substantial attention in recent years. Strong experimental data in SWE suggest that shear wave speed changes significantly due to the known acoustoelastic effect (AE). This presents both challenges and opportunities toward in vivo characterization of biological soft tissues. In this work, under the framework of continuum mechanics, we model a tissue-mimicking material as a homogeneous, isotropic, incompressible, hyperelastic material. Our primary objective is to quantitatively and qualitatively compare experimentally measured acoustoelastic data with model-predicted outcomes using multiple strain energy functions. Our analysis indicated that the classic neo-Hookean and Mooney-Rivlin models are inadequate for modeling the AE in tissue-mimicking materials. However, a subclass of strain energy functions containing both high-order /exponential term(s) and second-order invariant dependence showed good agreement with experimental data. Based on data investigated, we also found that discrepancies may exist between parameters inversely estimated from uniaxial compression and SWE data. Overall, our findings may improve our understanding of clinical SWE results.
\end{abstract}

Address all correspondence to:

Jingfeng Jiang, PhD

Dept. of Biomedical Engineering, Michigan Technological University

Minearl and Material Science and Engineering Building, Room 309

1400 Townsend Drive, Houghton MI 49931, USA

Email:jjiang1@mtu.edu

Phone: 906-487-1943

Fax: 906-487-1717

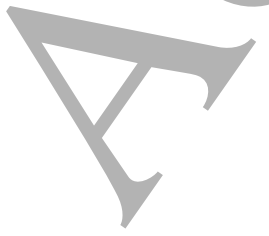




\section{To be submitted to Physics in Medicine and Biology}

\section{Introduction}

In the last decade, significant research efforts have been devoted to applying ultrasound(US) based elastographic techniques (Shiina et al., 2015) to non-invasively quantify viscoelastic properties of soft tissues in vivo. Among them, shear wave elastography (SWE) (Gennisson et al., 2013) characterizes tissue elasticity mainly based on quantification of propagating shear wave speed (SWS) inside the tissue of interest. Consequently, tissue elasticity can be inferred from a material model relating the elasticity to the SWS. Using acoustic radiation force (ARF) to excite soft tissues, Sarvazyan et al. (Sarvazyan et al., 1998) first demonstrated that small-amplitude (typically micrometers) shear waves could be generated and tracked using MR or optical scanners. Subsequently, other research groups (Nightingale et al., 2003, Bercoff et al., 2004) showed that both ARF pushing and ultrasonic tracking can be integrated into modern clinical ultrasound scanners. Promising clinical results have been published in the recent literature on the utility of SWE in the staging of liver fibrosis (Barr et al., 2015), breast lesion differentiation (Chang et al., 2013), estimations of muscle loading (Hug et al., 2015), evaluation of tendon damage (DeWall et al., 2014), and assessment of the risk of pre-term birth (Carlson et al., 2015). Successes of SWE have prompted releases of commercial SWE systems from major vendors (e.g. Siemens, GE, SuperSonic Imagine and Philips). Currently, SWE is frequently used as an adjunct to conventional US techniques in the routine clinical evaluation of soft tissue elasticity.

Continued developments of SWE techniques are ongoing. Among them, estimation of tissue hyperelastic parameters using the concept of acoustoelastic effect (AE) (Ogden, 2007) in conjunction with SWE data is particularly intriguing (Gennisson et al., 2007, Jiang et al., 2015a, Bernal et al., 2016, Aristizabal et al., 2018). If successful, this approach could offer a viable option to estimate mechanical properties of many in vivo biological tissues. The determination of mechanical properties of biological tissues has broad applications far beyond clinical diagnoses mentioned above. For instance, computer simulations of traumatic injury (e.g. traumatic brain injury owing to shocks or blast waves in sports) and virtual surgery require precise knowledge of the detailed mechanical parameters of tissues involved.

Among those reports (Gennisson et al., 2007, Jiang et al., 2015a, Bernal et al., 2016, Aristizabal et al., 2018), two AE formulations have been used to establish the relationship between the SWS and elastic constants. In the formulation in (Gennisson et al., 2007 ) is restricted to a strain energy function(SEF) that is a series expansion of the Green strain tensor and has been linearized in order to establish the relationship between SWS and elastic constants as a function of load (i.e. stress), making the analysis in principle limited to small strains. Under the framework of finite elasticity, an alternative formulation (Ogden, 2007) accepts any explicit definition of a SEF to determine SWS as a function of deformation. The latter formulation was first introduced in the context of SWE by Jiang et al. (Jiang et al., 2015a) and is here after referred to as Ogden's formulation. Because the latter formulation can be readily applied to conven- 


\section{To be submitted to Physics in Medicine and Biology}

tional hyperelastic constitutive models over their full elastic range, it has been adopted for this study.

Toward this end, our primary objective is to evaluate several commonly-used and representative hyperelastic SEFs in terms of their ability to represent AE using SWS data (hereafter referred to as AE-SWS data) obtained from tissue-mimicking materials. This is the first study where SEFs are compared against each other to investigate their fitness to AE-SWS data. In order to do so, we adopted the small-on-large acoustoelastic analysis framework (Norris, 2007, Ogden, 2007) and modeled the medium as a homogeneous, isotropic and incompressible, hyperelastic material. Such an evaluation study will enable us to determine whether or not an "optimal" SEF exists. As of now, the choice of SEF is not well understood and its implication for the estimation of nonlinear elastic parameters is not fully investigated. The secondary objective of this study is to investigate the consistency in terms of estimation of nonlinear elastic parameters between AE-SWE and conventional uniaxial data (stress-strain data).

Our analysis will first be applied to previously reported AE-SWE data for a tissuemimicking phantom with the finite strain data required to apply the selected formulation. We will then apply our analysis to our own in-house experiments, which extends this formulation to three orthogonal shear wave propagation directions.

\section{Shear Wave Propagation in Finitely Deformed Solids}

The detailed derivation of the method has been well established by Odgen (Ogden, 2007) and thus, we omit certain details.

\subsection{Basics in Finite Elasticity}

Our solid is initially at rest in the initial reference geometry $\left(\mathcal{B}_{r}\right)$. By stressing the solid, a finitely deformed geometry $(\mathcal{B})$ can be obtained. A point initially at $X$ in $\mathcal{B}_{r}$ is at $x=\chi(X)$ in $\mathcal{B}$, where $\chi$ is a function for a one-to-one mapping from $\mathcal{B}_{r}$ to $\mathcal{B}$; see Fig 1 . The deformation tensor $F$ describing the mapping is defined below,

$$
F=\frac{\partial \chi}{\partial X}
$$

where $F_{i j}=\frac{\partial x_{i}}{\partial X_{j}}$. In order to model mechanical behaviors of the solid, SEFs are often formulated in various forms based on left/right Cauchy-Green tensor or its invariants and principal stretch. Those SEFs fall into the category of phenomenological constitutive modeling. The right Cauchy-Green tensor is defined below as

$$
C=F^{T} F
$$

Its three invariants can be subsequently defined as follows

$$
I_{1}=\operatorname{tr}(C), I_{2}=1 / 2\left[I_{1}^{2}-\operatorname{tr}\left(C^{2}\right)\right], I_{3}=\operatorname{det}(C)
$$




\section{To be submitted to Physics in Medicine and Biology}

where $\operatorname{det}()$ and $\operatorname{tr}()$ are the determinant and trace operations respectively. In the case of incompressibility, $I_{3}=1$. The three invariants above can also be expressed in terms of the principle stretches $\left(\lambda_{1}, \lambda_{2}\right.$ and $\left.\lambda_{3}\right)$ below,

$$
\begin{aligned}
& I_{1}=\lambda_{1}^{2}+\lambda_{2}^{2}+\lambda_{3}^{2} \\
& I_{2}=\lambda_{1}^{2} \lambda_{2}^{2}+\lambda_{1}^{2} \lambda_{3}^{2}+\lambda_{2}^{2} \lambda_{3}^{2} \\
& I_{3}=1=\lambda_{1}^{2} \lambda_{2}^{2} \lambda_{3}^{2}
\end{aligned}
$$

where a principal stretch refers to the ratio of length change along one principal axis between $\mathcal{B}_{r}$ and $\mathcal{B}$.

Given an arbitrary SEF, $W$, Eqn. (7) below introduces the elasticity tensor $\Xi$ which is a 4th rank tensor.

$$
\Xi_{\alpha i \beta j}=\left(\frac{\partial^{2} W}{\partial F_{\alpha i} \partial F_{\beta j}}\right)
$$


To be submitted to Physics in Medicine and Biology

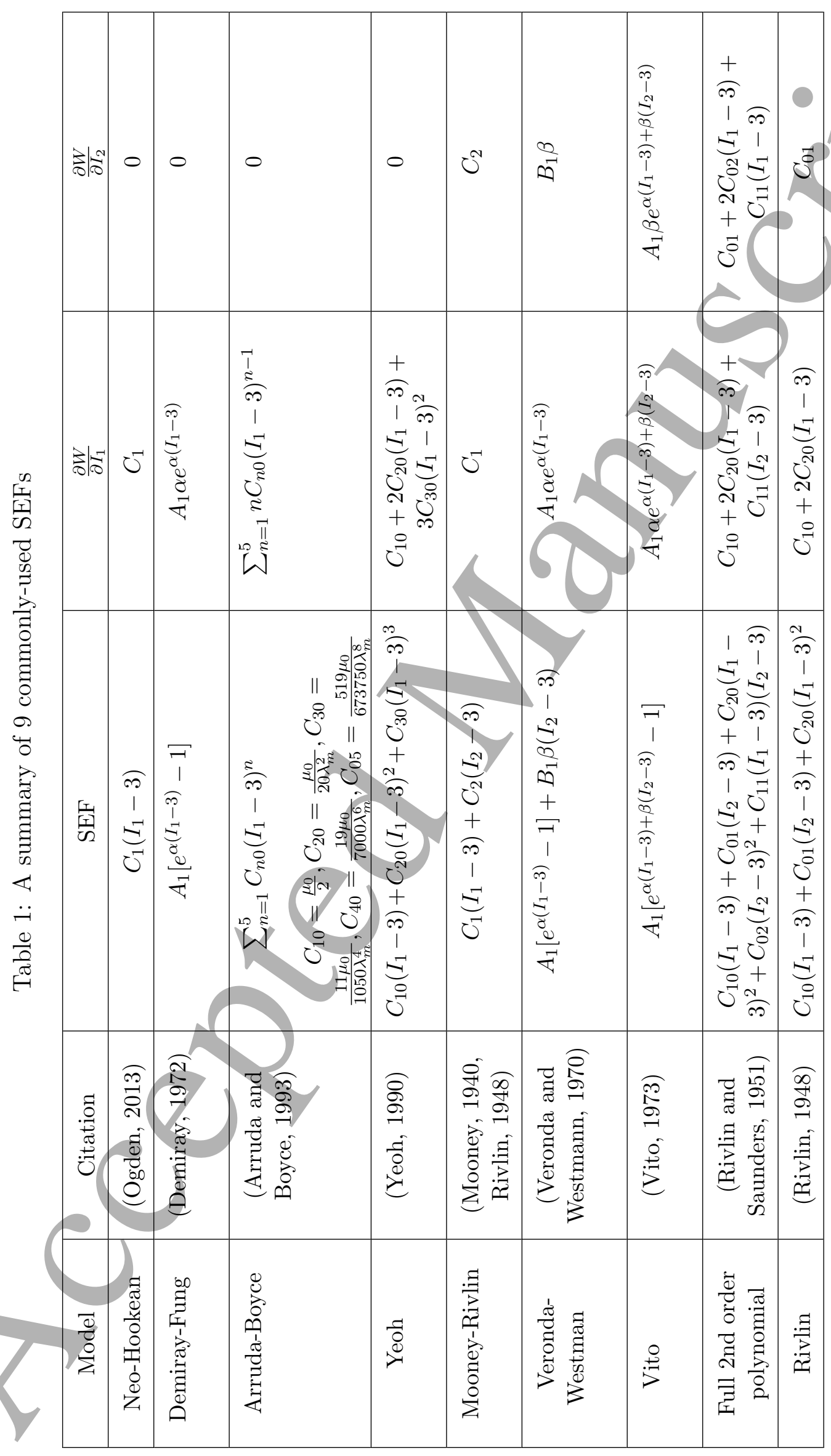


To be submitted to Physics in Medicine and Biology

Invariant-based SEFs are popular because they offer simpler mathematical forms and have few parameters. Table 1 lists nine (9) commonly used SEFs of this kind and those SEFs will be evaluated in this study.

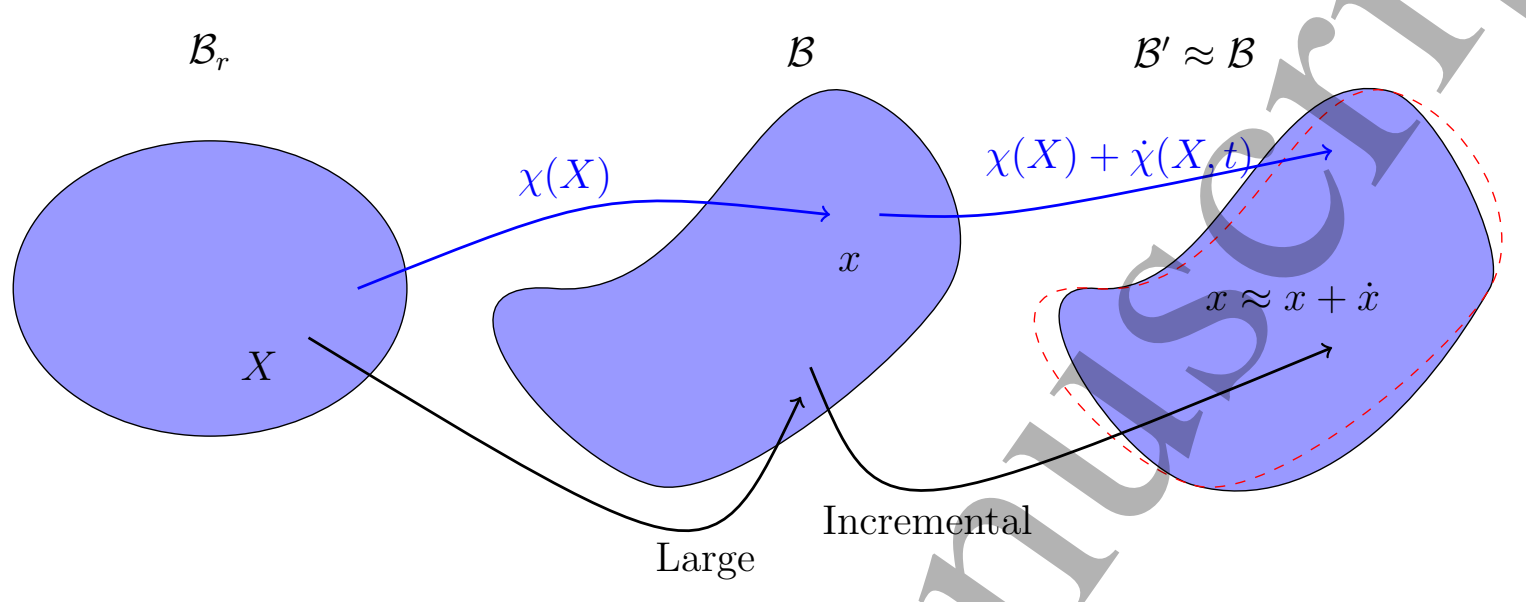

Figure 1: An illustration of successive deformation patterns occuring in the soft solid: first a large static homogeneous deformation and then an incremental deformation due to the propagation of a small-amplitude shear wave.

\subsection{Wave Equation in Finitely Deformed Media}

When a shear wave travels through a finitely deformed medium, the wave causes a small incremental deformation. This treatment of acoustoelasticity is known as small-on-large acoustoelasticity (Ogden, 2007) as follows,

$$
x=\chi(X) \rightarrow x+\dot{x}=\chi(X)+\dot{\chi}(X, t)
$$

where a dot denotes an incremental quantity and the a right arrow indicates "is incremented to". Thus, the incremental deformation tensor $\dot{F}$ can be expressed as follows,

$$
\dot{F}=\frac{\partial \dot{\chi}}{\partial X}
$$

where $\dot{F}_{i j}=\frac{\partial \dot{x}_{i}}{\partial X_{j}}$. In Eqn. (9), the above gradient is defined relative to the undeformed reference configuration $\left(\mathcal{B}_{r}\right)$. Practically, it would be more useful to use displacements formulated relative to the deformed configuration $(\mathcal{B})$ in the framework of AE-SWE. This is because ultrasonically measured displacement and velocity data are relative to the finitely deformed configuration. To proceed, we consider incremental displacement induced by the shear wave in the Eulerian frame, $u(x, t) \equiv \dot{\chi}(x, t)$, as formulated by (Ogden2007), which is

$$
u(x, t)=u(\chi(X), t)=\dot{\chi}\left(\chi^{-1}(x)\right)
$$

The incremental deformation tensor relative to the deformed configuration $\Gamma$ then becomes 
To be submitted to Physics in Medicine and Biology

$$
\Gamma=\frac{\partial \dot{\chi}}{\partial x}=\frac{\partial \dot{\chi}}{\partial X} \frac{\partial X}{\partial x}=\dot{F} F^{-1}
$$

Applying the incompressiblilty condition to Eqn. (11) yields

$$
\operatorname{div}(u)=\operatorname{tr}(\Gamma)=0
$$

where $\operatorname{div}()$ stands for the divergence operation.

It has been demonstrated that the incremental analogue to the Cauchy stress, denoted here by $\dot{\sigma}$, can be defined in terms of $\Gamma$ as follows (Ogden, 2007),

$$
\dot{\sigma}=\Xi_{0} \Gamma+p \Gamma-\dot{p} I
$$

Here $p$ and $\dot{p}$ are Lagrangian multipliers that enforce incompressibility from the finite and infintesmal deformations respectively. Likewise, $\Xi_{0}$ is the elasticity tensor relative to the deformed configuration $(\mathcal{B})$, which has the following components

$$
\Xi_{0 p i q j}=J^{-1} F_{p \alpha} F_{q \beta} \Xi_{\alpha i \beta j}
$$

Neglecting body forces, the incremental equations of motion relative to $\dot{\sigma}$ and $u$ are then

$$
\operatorname{div}(\dot{\sigma})=\rho u_{, t}
$$

Substitution of Eqn. (13) into Eqn. (15) produces)

$$
\Xi_{0 p i q j} u_{j, p q}-\dot{p}_{, i}=\rho u_{i, t t}
$$

Equation (16) is the wave equation for a statically pre-deformed incompressible, isotropic medium, which will be used for subsequent analyses of the AE effect.

Under a 2D plane wave assumption, Ogden solved the wave equation developed in Eqn. (16). The SWS $c$ for a shear wave propagating within a plane defined by two principle axes of the stretches $\lambda_{i}$ and $\lambda_{j}$ is (Ogden, 2007),

$$
\begin{aligned}
& \rho c^{2}=(\alpha+\gamma-2 \beta) \cos ^{4}(\theta)+2(\beta-\gamma) \cos ^{2}(\theta)+\gamma, \\
& \alpha=\Xi_{0 j i j i}, \quad \gamma=\Xi_{0 i j i j}, \quad 2 \beta=\Xi_{0 i i i i}+\Xi_{0 j j j j}-2 \Xi_{0 i i j j}-2 \Xi_{0 i j j i}
\end{aligned}
$$

where $\rho$ is the mass density and $\theta$ is the angle between the propagation direction and the principle axis of $\lambda_{i}$. No summation is implied by the repeated indices in Eqn. (17). For an isotropic solid, $\Xi_{0}$ can be described to facilitate its use in conjunction with invariant-based SEFs by (Ogden, 2007),

$$
\Xi_{0 l k l k}=\lambda_{l}^{2} \frac{\lambda_{l} \frac{\partial W}{\partial \lambda_{l}}-\lambda_{k} \frac{\partial W}{\partial \lambda_{k}}}{\lambda_{l}^{2}-\lambda_{k}^{2}} \quad l \neq k \quad \lambda_{l} \neq \lambda_{k}
$$

Combining basic expressions of invariants from Eqn. (4) and (5) with Eqn. (19) produces

$$
\Xi_{0 l k l k}=2 \lambda_{l}^{2}\left(\frac{\partial W}{\partial I_{1}}+\frac{\partial W}{\partial I_{2}} \lambda_{l}^{-2} \lambda_{k}^{-2}\right)
$$



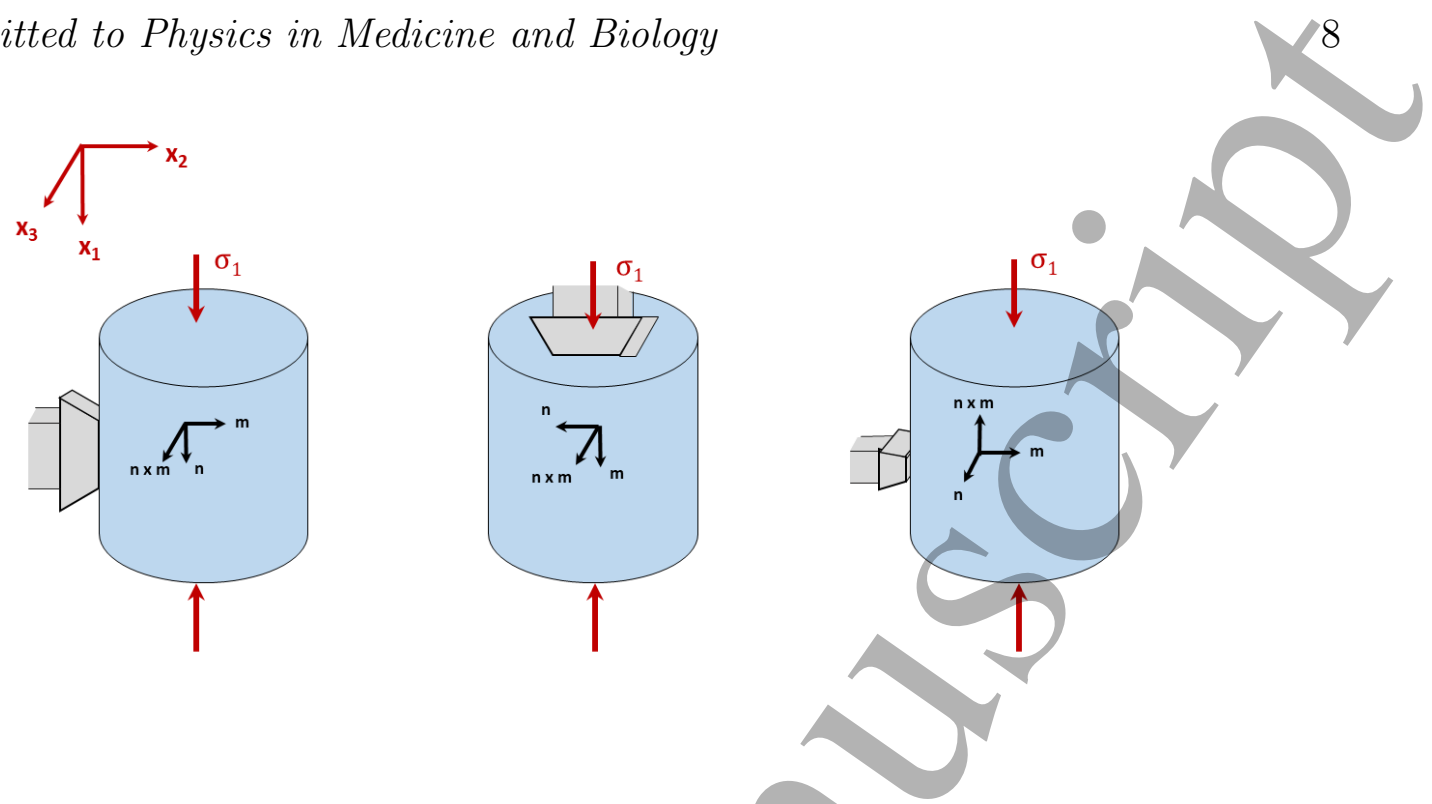

Figure 2: A plot showing three possible configurations generating AE-SWE data in a phantom experiment where the phantom is under an unaxial stress $\sigma_{1}$. The three resulting shear waves are designated as 12, 21, and 23 from the left to the right, respectively. Thus, corresponding SWS values are designated as $c_{12}, c_{21}$ and $c_{23}$ in this article, respectively. $\mathrm{m}$ and $\mathrm{n}$ stand for polarization and shear wave propagation directions, respectively.

\subsection{The Role of the SEF in Analysis of AE-SWE Measurements}

In this section, the theoretical framework laid out in the previous sections will be developed into equations from which AE-SWE data (both published and in-house phantom data) can be analyzed.

2.3.1. Considerations of AE-SWE Data Acquisition In the AE-SWE experiments reported in the literature (Jiang et al., 2015a,b, Gennisson et al., 2007, Urban et al., 2014), tissue-mimicking phantoms or ex vivo tissues were compressed along one direction. Particularly, in the work by Jiang et al. (Jiang et al., 2015a), the compression was applied by the face of the transducer and the off axis principle stretches were parameterized as $\lambda_{1}=\lambda, \lambda_{2}=\lambda^{-\xi}$, and $\lambda_{3}=\lambda^{-1(1-\xi)}$, where $\lambda$ is the stretch along the compression direction and $\xi$ is a parameter having a value from 0 to 1 . Note that this parameterization satisfies Eqn. (6) so that incompressibility is enforced.

Other experiments have considered SWE measurements from three orthogonal imaging planes (Gennisson et al., 2007, Urban et al., 2014) as illustrated in Fig. 2, though this has yet to be considered in the context Ogden's formulation and the large strain data required to do so (maximum compressive strain in the range of 25-55\%) is lacking. The acoustoélastic equations for these three planes are produced by setting the indices in Eqn. (17) to coincide with the relevant plane (i.e. 12, 21 or 23) and setting $\theta$ to zero. 
To be submitted to Physics in Medicine and Biology

This produces the following three equations:

$$
\begin{aligned}
& \rho c_{12}^{2}=\Xi_{01212}=2 \lambda^{2} \frac{\partial W}{\partial I_{1}}+2 \lambda^{2 \xi} \frac{\partial W}{\partial I_{2}} \\
& \rho c_{21}^{2}=\Xi_{02121}=2 \lambda^{-2 \xi} \frac{\partial W}{\partial I_{1}}+2 \lambda^{-2} \frac{\partial W}{\partial I_{2}} \\
& \rho c_{23}^{2}=\Xi_{03232}=2 \lambda^{-2+2 \xi} \frac{\partial W}{\partial I_{1}}+2 \lambda^{2 \xi} \frac{\partial W}{\partial I_{2}}
\end{aligned}
$$

Of note, by setting $\xi=0.5$, Eqns. (21-23) represent SWS within three orthogonal planes under the uniaxial loading case, i.e. $\lambda_{2}=\lambda_{3}$.

2.3.2. The Role of SEFs A wide variety of functional forms for $W$ have been proposed for modeling biomaterials as an isotropic, hyperelastic, incompressible solid. A recent review of these various models and their applications can be found elsewhere (Wex et al., 2015). Below we briefly discuss some representative SEFs in two categories: polynomial and exponential forms.

Polynomial Form SEFs of the polynomial form have been applied to mechanical test data of breast tissue (Joseph and Abbas, 2009). The general representation of $W$ in terms of a polynomial series would be (Rivlin and Sáunders, 1951)

$$
W=\sum_{i+j=1}^{N} C_{i j}\left(I_{1}-3\right)^{i}\left(I_{2}-3\right)^{j}
$$

A variety of common models can be arrived at in terms of this series. It is easy to see both the classic Mooney-Rivlin (setting $N$ to 1 ) and Neo-Hookean (eliminating $I_{2}$ ) models are special cases of Eqn. (24). Further expansion of the series to include higher order terms is typical when it is desired that the model captures large strain behaviors. However, inclusion of all possible polynomial coefficients in Eqn.( 24) at higher values of $N$ produces infeasible numbers of material parameters. Thus, the order of the polymonial equation has to be reasonably small for practical reasons. As a result, it is typical for certain coefficients to be neglected in polynomial models of higher orders. For instance, the Yeoh model (Yeoh, 1990) and the polynomial representation of the Arruda-Boyce(Arruda and Boyce, 1993) model retains only the coefficients exclusive to $I_{1}$ (i.e. $C_{i 0}$ ). Table 1 lists several variants of Eqn. (24).

Exponential Form Models in this category first emerged as an attempt to incorporate the exponential solution to Fung's law (Fung, 1967) into a 3D continuum framework (Demiray, 1972). The details of the mathematical form of these models have typically been arrived at by guess work. More recently, a general expression for these kinds of models has been formulated (Mansouri and Darijani, 2014). With some adjustments, this general expression can be written as

$$
W=\sum_{k}^{N} A_{k}\left(e^{f\left(\lambda_{1}, \lambda_{2}, \lambda_{3}\right)}-1\right)+\sum_{k}^{N} B_{k}\left(e^{g\left(\lambda_{1}, \lambda_{2}, \lambda_{3}\right)}-1\right)
$$




\section{To be submitted to Physics in Medicine and Biology}

Here, $A_{k}$ and $B_{k}$ are material parameters having units in kiloPascals, and $f$ and $g$ are unitless functions that define the nonlinear elastic behavior of the model. A prior publication (Mansouri and Darijani, 2014) outlined the mathematical restrictions on $f$ and $g$, as well as a wealth of permissible functions that meet these restrictions. Virtually all of the exponential models that have been put to use in the soft tissue literature can be represented from Ean. (25) when $N$ is set to 1 and $f$ and $g$ are composed of a suitable combination of $\alpha\left(I_{i}-3\right), \alpha \ln \left(I_{i} / 3\right)$, and 0 , where $\alpha$ is a unitless parameter. For instance, the Veronda-Westman model (Veronda and Westmann, 1970), which is in common use in strain elastography (Shiina et al., 2015), is produced from Eqn. (25) when $f$ is set to $\alpha\left(I_{1}-3\right)$ and $g$ is set to $\beta \ln \left(I_{2} / 3\right)$. Note that the Mooney-Rivlin and Neo-Hookean models can also be produced by Eqn. (25) by setting $f$ to $\alpha \ln \left(I_{1} / 3\right)$ and $g$ to either $\beta \ln \left(I_{2} / 3\right)$ or 0 , respectively. In this sense, the Mooney-Rivlin and Neo-Hookean models can be thought of as a special case of either Eqn. (24) or (25).

A Summary Statement Table 1 lists the exponential and polynomial models that were evaluated in this study for their fitness in terms of analysis of AE. As established by Eqns. (21-23), different response functions $\frac{\partial W}{\partial I_{1}}$ and $\frac{\partial W}{\partial I_{2}}$ induced by different SEFs lead to different behaviors among acquired AE-SWE data. In Table 1, response functions $\frac{\partial W}{\partial I_{1}}$ and $\frac{\partial W}{\partial I_{2}}$ are also tabulated. By having the response functions tabulated as they are, they can readily be applied to $\mathrm{AE}$ analysis and interpret experimentally obtained AE-SWE data below.

\section{Methods and Materials}

\subsection{Description of Tissue-mimicking Phantom Experiments}

3.1.1. Experiments Reported by Jiang et al. (2015a) SWE measurements reported in Jiang et al. were collected from a phantom constructed from cryogenically crosslinked $10 \%$ polyvinal alcohol (PVA), and $3 \%$ Sigmacell was used as the source of acoustic scattering. The biaxial deformation of the phantom was parameterized by measuring the relative motion of three wires embedded in the phantom and computing value for $\xi$ from these measurements. For our model fitting, we use the same biaxial parameterization reported (i.e. $\xi=0.2$ ). SWE measurements were performed using the Supersonic Shear Imaging (SSI) technique using a vendor-supplied on-screen software package (SuperSonic Imagine Inc., Aix-en-Provence, France). After SWE measurements, the phantom was cut into smaller samples for uniaxial tensile tests to obtain stress-strain data. More details can be found elsewhere (Jiang et al., 2015a).

3.1.2. In-house Experiments In-house data were collected from two cylindrical phantoms composed of $5 \%$ and $10 \%$ by weight of cross-linked gelatin. The phantom construction process mostly followed that of Hall et al. (Hall et al., 1997).Cellulose particles 
(SigmaCell; Sigma-Aldrich Inc., MI, USA) were then mixed into the gelatin solution to $3 \%$ by weight and were the source of acoustic scattering. A cross-linker (formalin) was also added at $0.047 \mathrm{~g}$ of formaldehyde per a gram of dry weight gelatin. The resulting gelatin solutions were congealed in silicon molds for cylindrical phantom shape $(90 \mathrm{~mm}$ height and $80 \mathrm{~mm}$ diameter).

To apply a uniaxial deformation to the phantoms during SWE measurements, the phantom was placed between a lab jack and a stationary acrylic compression plate. During measurements, a scale was placed underneath the lab jack to measure the load applied to the phantom, while the lab jack was raised to deform the phantom and digital calipers were used to record the height of the phantom. Then a single ultrasound transducer (L7-4 linear array transducer, ATL, Phillips Inc., WA) held stationary in one of the three orientations illustrated in Fig. 3. As illustrated, measurements collected on the side of the phantom were made at approximately $28 \mathrm{~mm}$ from the top of the phantom and the measurement made at the top of the phantom was collected through an opening cut from the acrylic plate. A research ultrasound system (V1 system, Verasonics Inc., WA) equipped with the probe was used to obtain SWE measurements. The Verasonics system was first used for the creation of one single push-pulse excitation followed by ultrafast imaging of the resulting waveforms. A published Fourier domain shift-matching (FDSM) method (Rosen and Jiang, 2018) was used to estimate SWS. Both the SWE data acquisition, the FDSM method and its validation can be found in our previous publication (Rosen and Jiang, 2018).

A maximum of $40 \%$ compression was applied to each phantom with a load increment of $2.75 \%$ compression. In each orientation, the compression load stopped once the top of the lab jack was nearly in contact with the transducer when the transducer was applied to the side of the phantom image (i.e. $c_{12}$ image plane in Fig. 3). Following the initial SWE measurement, the deformation of the phantom was incremented by adjusting the height of the lab jack. Once the reading on the scale reached steady-state, the load, phantom height, and SWE measurements were recorded at the new deformation level. In each orientation, the phantom was then decompressed to zero load after reaching its maximal compression. Then, the transducer was adjusted to the next orientation. Measurements were collected in the order $c_{12}, c_{23}, c_{21}$. Regardless of the transducer orientation, the distance between the focus of the pushing-pulse and the transducer face was set to $19.7 \mathrm{~mm}$. Both phantoms were stable under load repetitions because variations in the initial phantom heights were less than $1.1 \%$ and $0.1 \%$ for the $5 \%$ and $10 \%$ gelatin phantoms, respectively. The height variation was measured by calculating the maximum difference as a percentage of the mean phantom height. During the SWE experiments, stress and strain values were converted from the above-mentioned load and height readings. 
To be submitted to Physics in Medicine and Biology
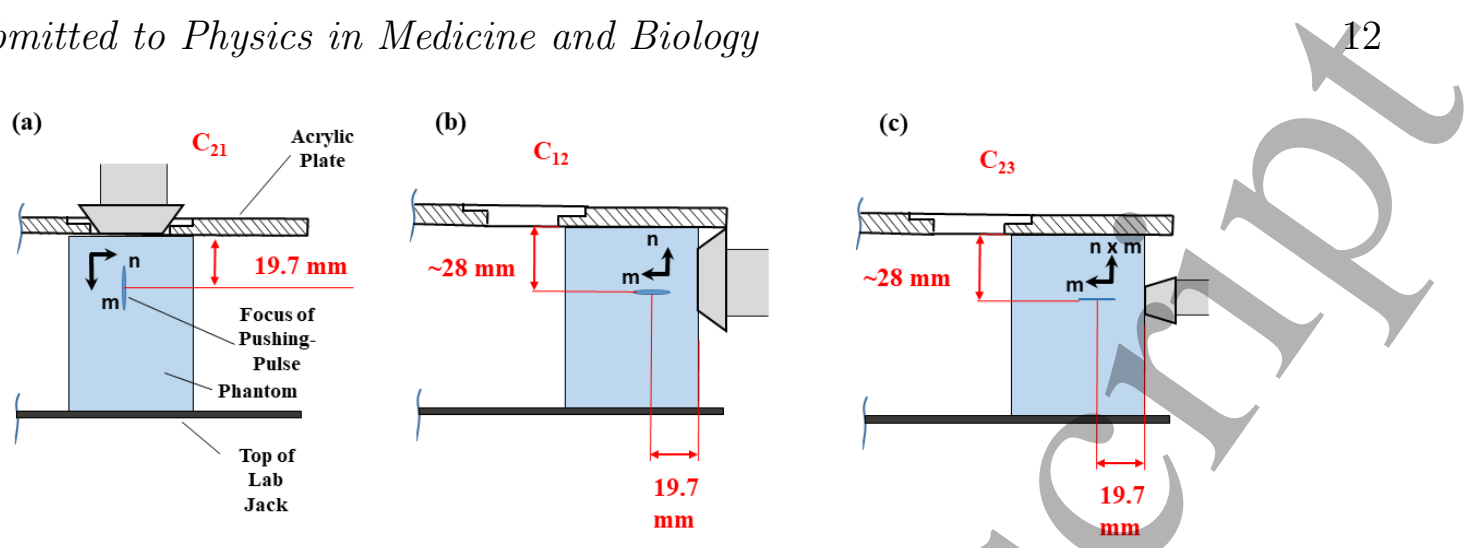

Figure 3: Cross-sectional illustration of the gelatin phantom experiments (not to scale). For the measurements collected in the $c_{21}$ orientation (a), the phantom was placed underneath an opening in the acrylic plate that allowed for imaging from the top of the phantom. For $c_{12}$ (b) and $c_{23}$ (c) orientations, the phantom was placed underneath a flat portion of the acrylic plate. The vectors $\mathrm{n}$ and $\mathrm{m}$ denote the direction of shear wave propagation and polarization, respectively.

\subsection{Data Analysis}

SWE and stress-strain data collected from the literature (Jiang et al., 2015a) was digitized using a free online graph digitizer $\ddagger$ (WebPlotDigitizer v4.1, Automeris LLC). In-house acquired using methods described/above were all stored in a computer. Parameters related to 9 different SEFs (see Table 1) were obtained by mathematical optimization. More specifically, SWE and uniaxial stress-strain data were used to fit respective SEFs by minimizing the sum of squared residuals between experimentally obtained and model predicted values. This process was accomplished by using the "fminunc" function in MATLAB (Version 2016a, Mathworks Inc., Natick, MA). The starting parameters for each model was set to correspond to Neo-Hookean material behavior. That is, all $I_{2}$ dependent parameters were started at 0 and higher-order or exponential strain stiffening terms set at or near their non-stiffening limit. During model fitting of SWE measurements, models were fixed at zero strain to the mean SWE measurement at no deformation.

To assess the fitness/merits of each of $9 \mathrm{SEFs}$, the root mean squared residual was calculated as follows:

$$
R M S R=\sqrt{\frac{\sum_{i=1}^{n}\left(\hat{d_{m}}-d_{e}\right)^{2}}{n}}
$$

Here $d$ stands for SWE or mechanical testing data, subscripts $m$ and $e$ denote the individual model-predicted and experimentally-obtained SWS or mechanical testing estimates, respectively. In Eqn. (26), $n$ is the number of experimentally obtained SWS or mechanical testing estimates .

t https://automeris.io/WebPlotDigitizer 


\section{Results}

\subsection{Reanalysis of Data from Jiang et al. (2015a)}

Model fitting of AE-SWE data from a published article (Jiang et al., 2015a) are plotted in Fig. 4. In order to make the plot more readable, the hyperelastic SEFs are separated into those that are functions of only $I_{1}$ (Fig. 4a) and those that are functions of both $I_{1}$ and $I_{2}$ (Fig. 4b). The motivation for this separation will be more apparent when considering the multiple orientations considered in the in-house data. From these results, it can be seen that all model fits incorporating second-order polynomial terms or exponential strain stiffening matched AE-SWE data well. In contrast, the neo-Hookean and Mooney-Rivlin models produced comparatively poor fits to the observed AE-SWE data.

Hyperelastic parameters were also obtained by using uniaxial tension data. Fig. 5 overlays fitted tensile stress-strain curves with experimental ones (Jiang et al., 2015a). It can be seen that the fitted tensile stress-strain curves for all models except the neoHookean and Mooney-Rivlin models tracked the tensile stress-strain data within one standard deviation.

Estimated hyperelastic parameters for all 9 SEFs (see Table 1) are displayed in Table 2 for both AE-SWE data and uniaxial stress-strain data fitting. It is interesting to note that the resulting parameters showed variable agreement between estimates from AESWE data and uniaxial tensile test data. Particularly, little consistency was found when the Mooney-Rivlin model was used.

\subsection{In-House Data}

Fig. 6 shows the AE-SWE results and model fitting of SEFs for the $5 \%$ and $10 \%$ gelatin phantoms. In contrast to the previous data set, when the models were fitted to three wave orientations, a clear distinction in the performance within the 2ndorder/exponential models is apparent. In particular, the models which lack $I_{2}$ in their SEFs (Fig. 6) where unable to accommodate deviation between $c_{21}$ and $c_{23}$, whereas the model which did incorporate $I_{2}$ in the SEF were able to fit the two wave orientations just fine. This was the case even for the Yeoh model, which has the same number of parameters as the Vito, Veronda-Westmann and Rivlin models.

When considering the model estimated using uniaxial compression data collected from the $5 \%$ and $10 \%$ gelatin phantoms (Fig. 7), model's performance was comparable to what was observed in a prior publication(Jiang et al., 2015a). Note that, even though the Mooney-Rivlin model incorporates $I_{2}$ in its SEF, it was unable to fit the three wave orientations. This can be attributed to the absence of higher-order polynomial terms or exponential terms in its SEF. 
To be submitted to Physics in Medicine and Biology

\author{
To be submitted to Physics in Medicine and Biology
}

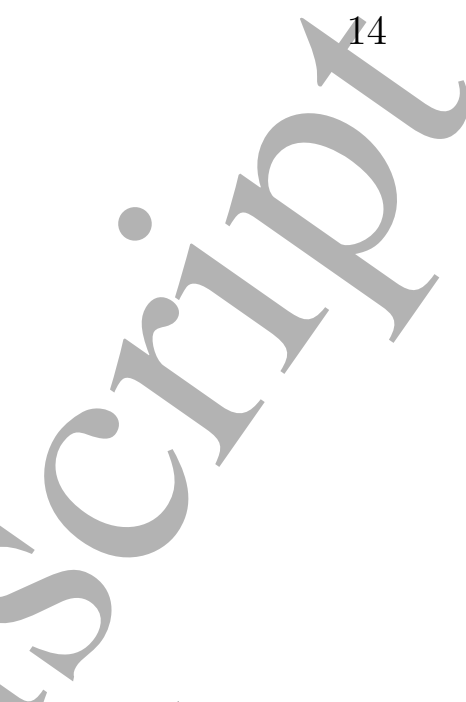
$\circ$

$\begin{array}{lll}20 & 10 \\ 0 & 10 & 10 \\ 0 & 0 & 7 \\ 0 & 0 & 0\end{array}$ 국

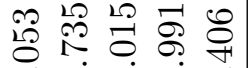

ํำ ลิ ลี กิ ते बं के

\title{
$\stackrel{8}{\stackrel{\overbrace{}}{\circ}}$
}

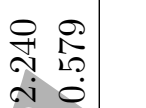
(2) is

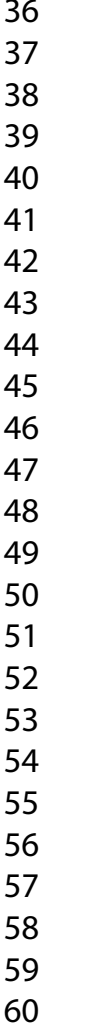

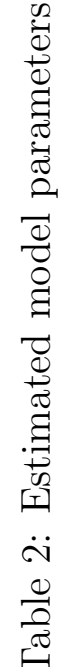

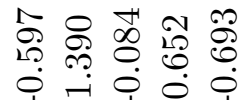

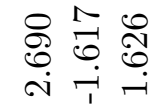
ने $\begin{array}{lllll}\dot{1} & - & 0 & 0 & 0 \\ 1 & 0 & 0\end{array}$ , 17

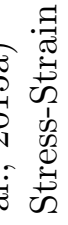
되

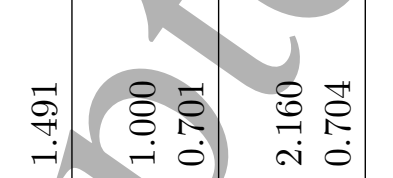

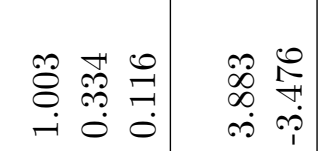
$\begin{array}{lll}2 & \Re & 2 \\ 8 & 8 & 0 \\ 0 & 0 & 1 \\ -1 & 0 & 0\end{array}$

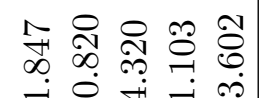

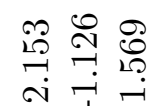
$(1)$

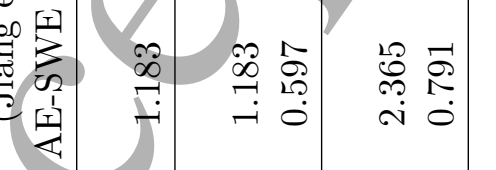

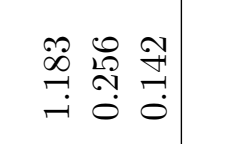

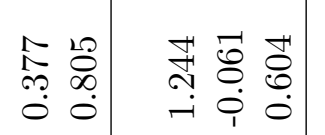

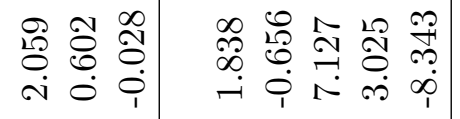

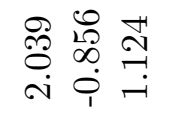

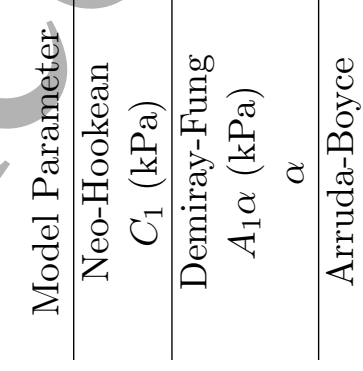
$\gtrless$

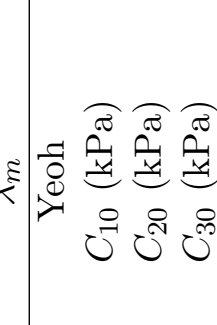

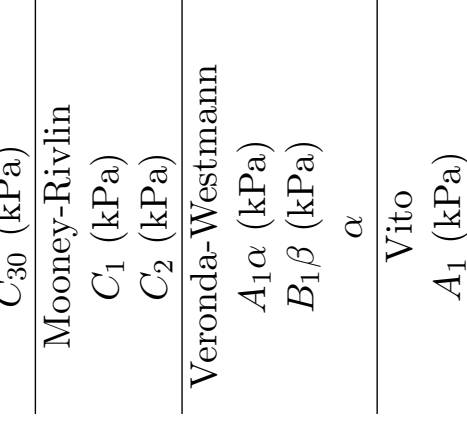

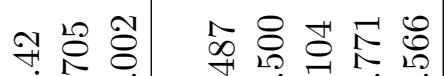

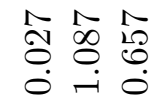

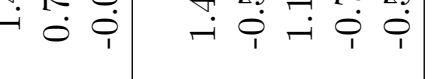
(2) i.

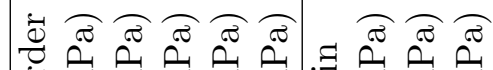

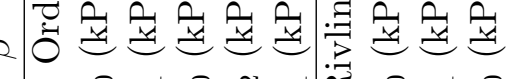
疍 
To be submitted to Physics in Medicine and Biology

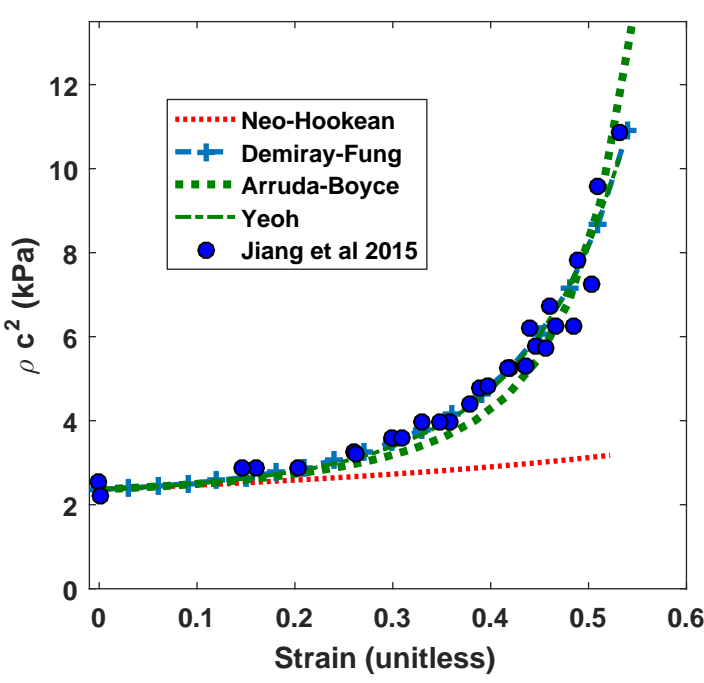

(a)

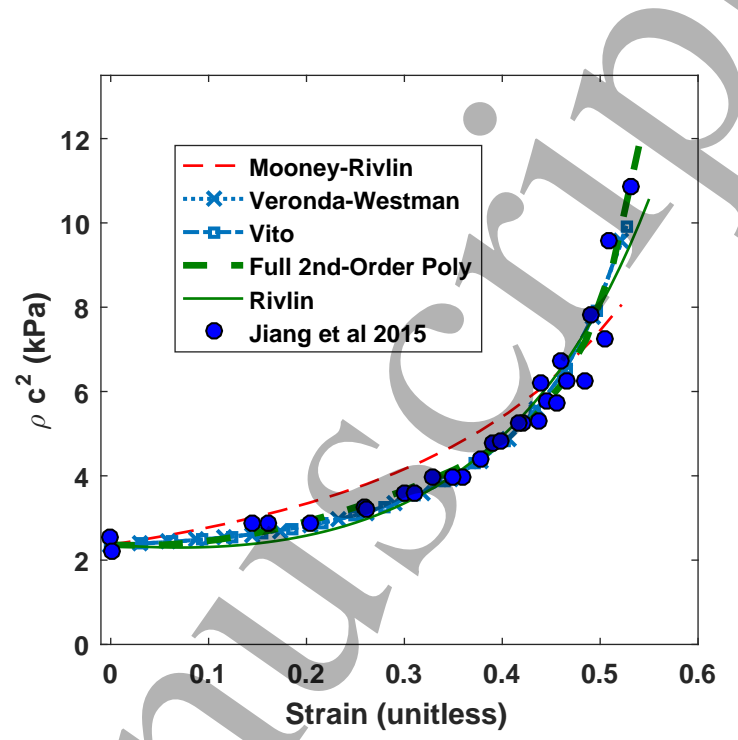

(b)

Figure 4: Fitting of SEFs listed in Table 1 to AE-SWE data from a published study (Jiang et al., 2015b). Curves are separated into models with no $I_{2}$ dependence (a) and models with $I_{2}$ dependence (b).

Table 3 tabulates RMSR values for all SEFs when they are fitted to both to AESWE and uniaxial tension/compression tests. These values support the observations made thus. In all cases, the Neo-Hookean and Mooney-Rivlin models produced the largest RSMR values. Additionally, the exponential and higher-order polynomial models that lack $I_{2}$ produced larger RSMR values for the two gelatin phantoms as compared to other RSMR values obtained from other models (1.1196-3.0428 kPa versus 0.1716$0.5817 \mathrm{kPa}$, respectively). Likewise, when uniaxial stress-strain data had been fitted, RMSR values were comparable. 
To be submitted to Physics in Medicine and Biology

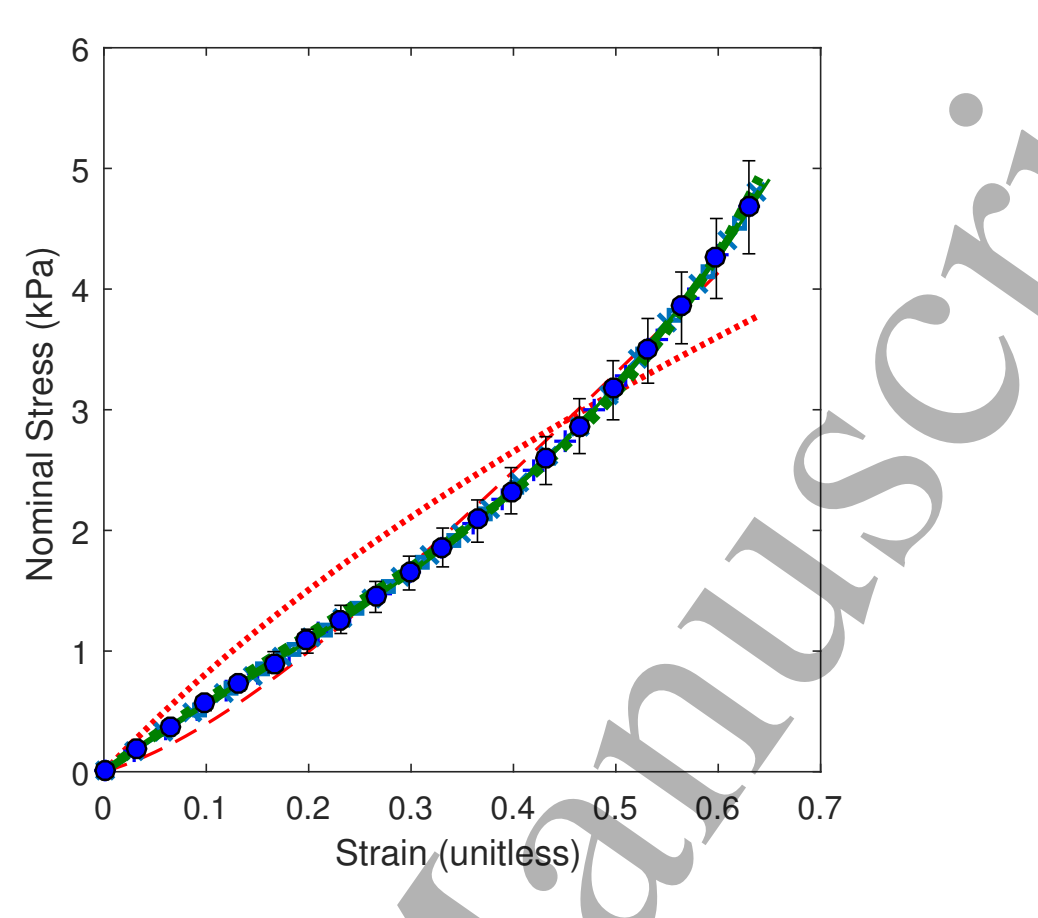

Figure 5: A plot comparing model-predicted mechanical behaviors under uniaxial tension with experimentally-measured ones (Jiang et al., 2015a). The solid blue circles denote the mean and one standard deviation of the nominal stress measured as a function of tensile strain. Figure legends were the same as those used in Fig. 4. 
To be submitted to Physics in Medicine and Biology

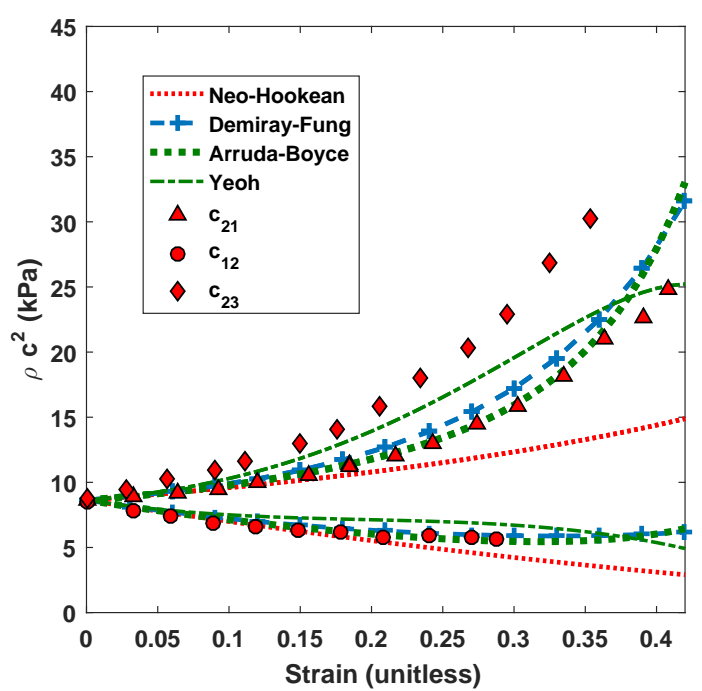

(a)

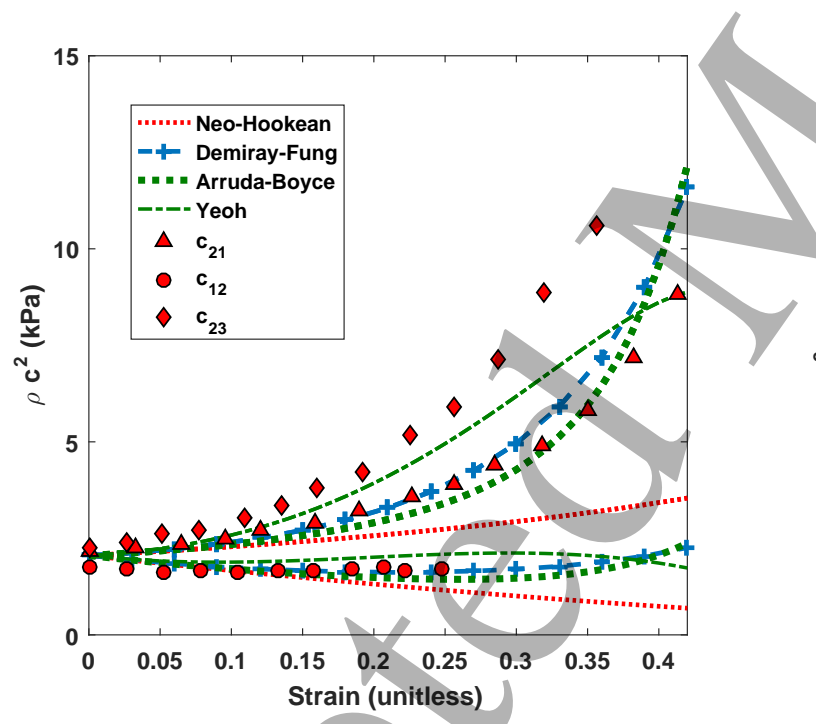

(c)

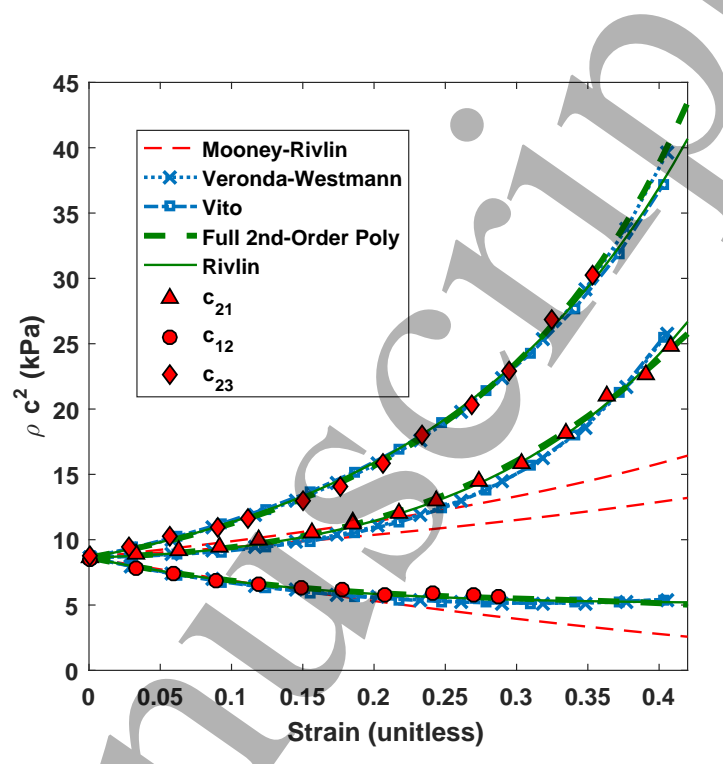

(b)

Figure 6: AE-SWE measurements collected from in-house $10 \%$ gelatin (top row) and $5 \%$ gelatin (bottom row) compressed elastography phantoms. Model fits are separated between models which do not include $I_{2}$ in their SEF (column 1) and models which do (column 2)

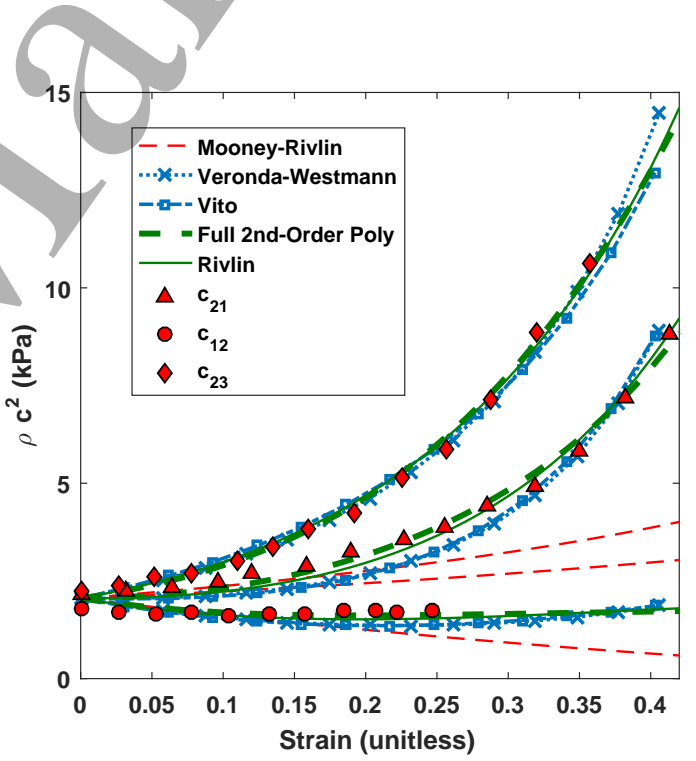

(d) 
To be submitted to Physics in Medicine and Biology

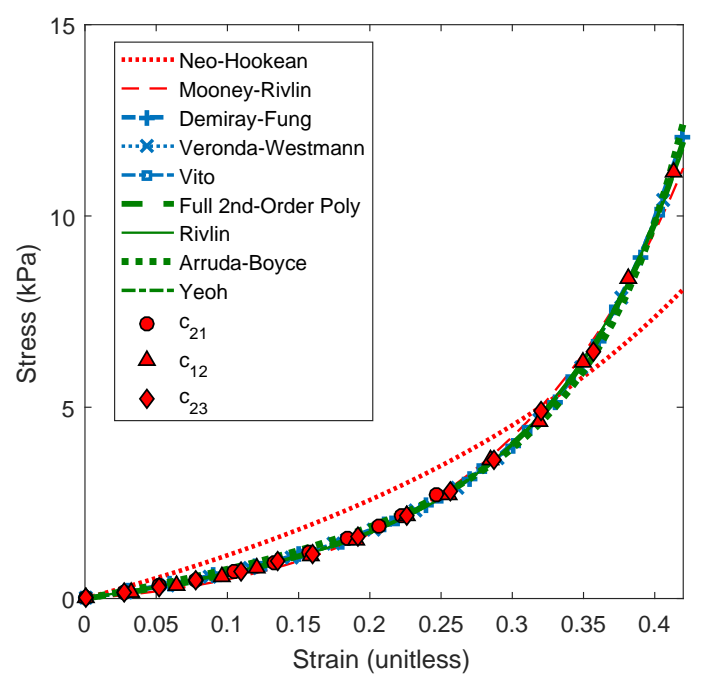

(a)

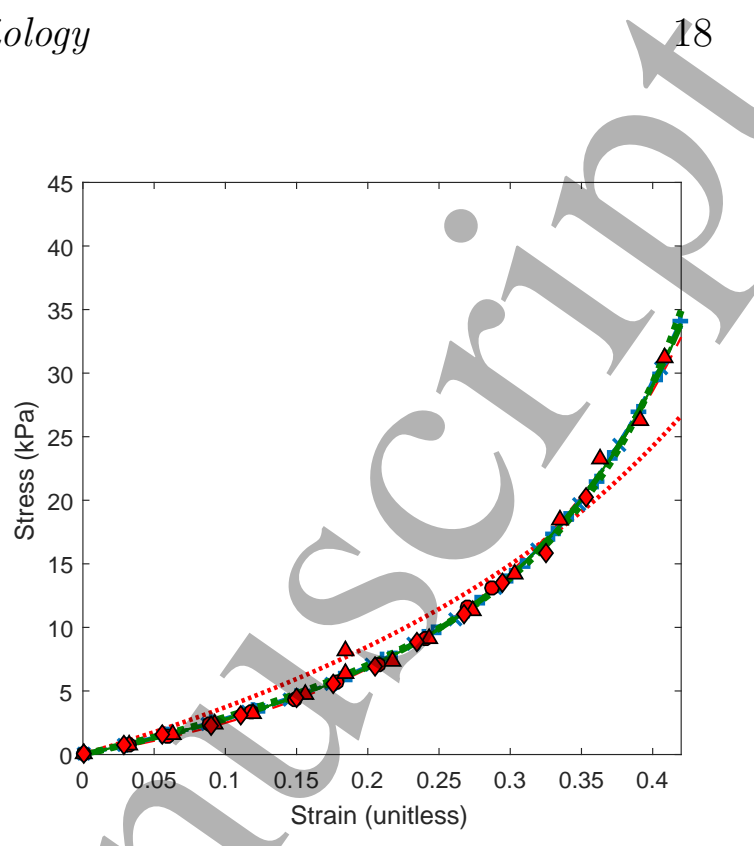

(b)

Figure 7: Combined compressive stress-strain data from $5 \%$ gelatin (a) and $10 \%$ gelatin (b) phantoms. Hyperlastic SEFs estimated from uniaxial compression data were used to predict compressive stress-strain curves and those predictions were overlaid with experimentally-obtained ones. 
To be submitted to Physics in Medicine and Biology

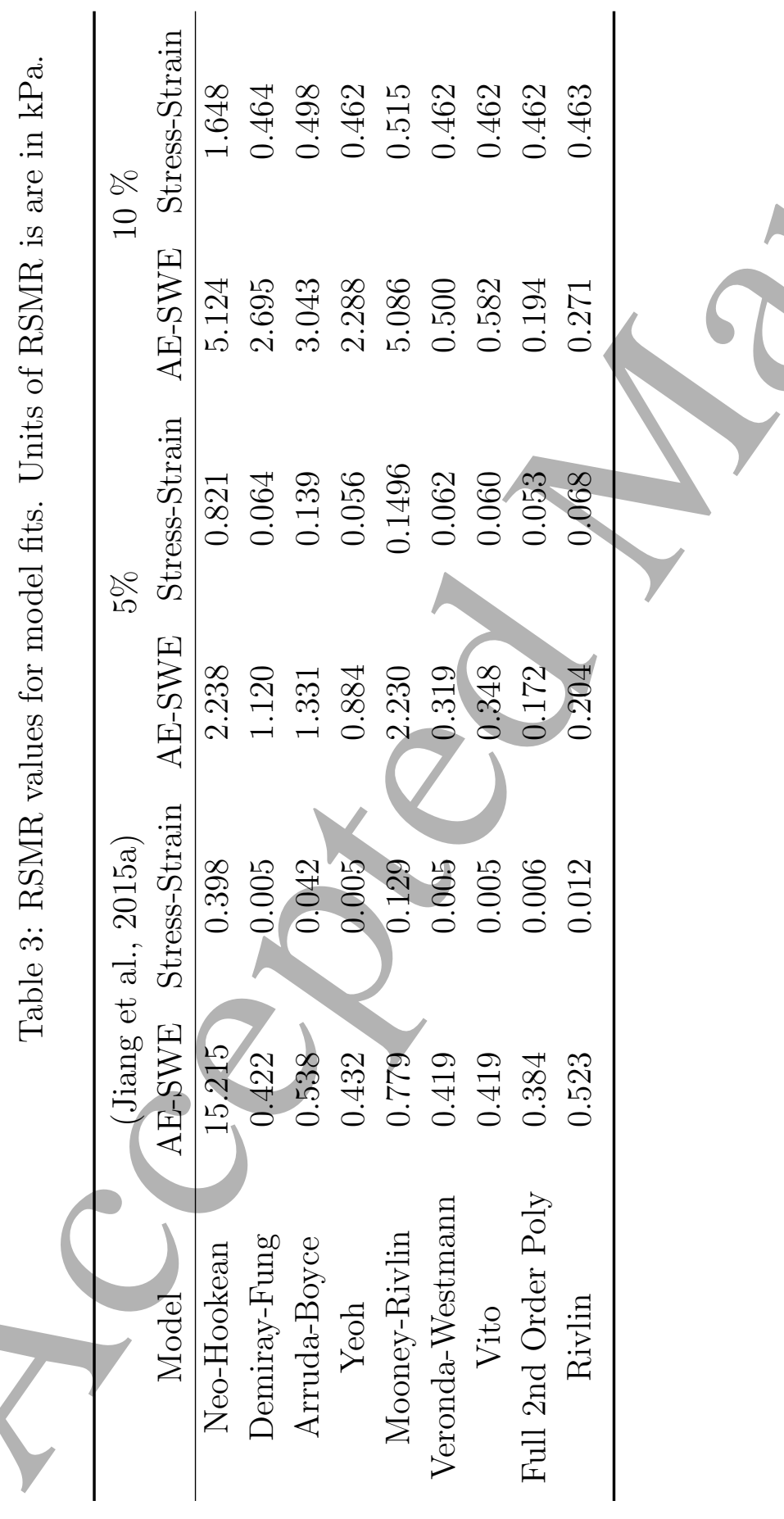




\section{To be submitted to Physics in Medicine and Biology}

Table 4 displays the fitted parameters produced from the separate fitting of the three imaging planes, as opposed to the combined fitting applied to produce the hyperelastic parameters reported in Table 2. In general, the estimated parameters tended to vary between fits to individual imaging planes as well as relative to the combined fitting, though the variation tended to depend on the model. For instance, the full second-order polynomial model tended to vary substantially within its terms, while the parameters in the Veronda-Westmann model were fairly similar to each other with exception to the fit of $c_{12}$ in the $5 \%$ gelatin phantom. Additionally, it is interesting to note that, for several of the models, fitting to $c_{21}$ alone tended to produce hyperelastic parameters similar to those produced from those obtained by fitting SEFs to uniaxial stress-strain data. For instance, in the $5 \%$ gelatin phantom, the parameter $\alpha$ in the Demiray-Fung model was similar when estimated from the uniaxial data and from $c_{21}$ alone (1.200 vs 1.167, respectively). In contrast, estimates from $c_{12}$ and $c_{23}$ (2.737 and 2.275 , respectively) show a more substantial difference to the uniaxial data. Clear exceptions to this observation included the Mooney-Rivlin, Vito and full second-order polynomial models.

\section{Discussion}

Although there are considerable interests in the estimation of hyperelastic material parameters (Samani and Plewes, 2004, Goenezen et al., 2012, Jiang et al., 2015a, Aristizabal et al., 2018), determination of such an appropriate SEF has not been fully explored. It is a challenge to introduce such a SEF in the elasticity imaging community because the SEF needs to meet the following two expectations: (1) capturing material behaviors and (2) having parameters that could provide insight to pathological and/or biological processors and are easy to understand by clinicians. It would be problematic if a selected SEF that is used to inversely estimate nonlinear mechanical properties cannot accurately represent mechanical behaviors. In regards to the first expectation, although biological tissue is likely to have more complex constitutive behavior than the tissue-mimicking phantom materials considered in this study, evaluating SEFs in these simple materials suggests some basic characteristics of SEFs to consider in AE-SWE investigations of complex biological media. Our overall observation (see Table 3) in this study is that SEFs containing the second invariant and second-order polynomial or exponential terms fitted experimental AE-SWE data better than those lacking one or both of these features. This difference can readily be observed in Fig. 6 and was the case even for the Yeoh model, which has the same number of parameters as the Veronda-Westmann model, but lacks second-invariant dependence. In particular, the full second-order polynomial SEF gave the best agreement with the experimental data, producing the lowest RMSR values. However, for fitting a single imaging plane, it produced comparatively less consistent results (Table 4). While the 3 parameter models, such as the Veronda-Westman or the Rivlin models, produced slightly higher RMSR than the full second-order polynomial model (e.g. in the \%10 gelatin phantom, 0.582 $\mathrm{kPa}$ and $0.271 \mathrm{kPa}$, respectively versus $0.194 \mathrm{kPa}$ ), they were generally able to represent 
To be submitted to Physics in Medicine and Biology

Table 4: A summary of hyperelastic parameters estimated only from AE-SWE data in our image plane defined in Fig. 2

\begin{tabular}{|c|c|c|c|c|c|c|}
\hline \multirow[b]{2}{*}{ Model Parameter } & \multicolumn{3}{|c|}{$5 \%$ Gelatin } & \multicolumn{3}{|c|}{$10 \%$ Gelatin } \\
\hline & $c_{21}$ & $c_{23}$ & $c_{12}$ & $c_{21}$ & $c_{23}$ & $c_{12}$ \\
\hline \multicolumn{7}{|l|}{ Neo-Hookean } \\
\hline$C_{1}(\mathrm{kPa})$ & 1.075 & 1.124 & 0.884 & 4.325 & 4.403 & 4.225 \\
\hline \multicolumn{7}{|l|}{ Demiray-Fung } \\
\hline$A_{1} \alpha(\mathrm{kPa})$ & 1.075 & 1.124 & 0.884 & 4.325 & 4.403 & 4.225 \\
\hline$\alpha$ & 1.167 & 2.275 & 2.737 & 0.726 & 1.686 & 0.823 \\
\hline \multicolumn{7}{|l|}{ Arruda-Boyce } \\
\hline$\mu$ & 2.15 & 2.248 & 1.768 & 8.651 & 8.805 & 8.451 \\
\hline$\lambda_{m}$ & 0.550 & 0.417 & 0.339 & 0.642 & 0.458 & 0.544 \\
\hline \multicolumn{7}{|l|}{ Yeoh } \\
\hline$C_{10}(\mathrm{kPa})$ & 1.075 & 1.124 & 0.844 & 44.325 & 4.403 & 4.225 \\
\hline$C_{20}(\mathrm{kPa})$ & 0.624 & 2.458 & 2.019 & 1.371 & 6.687 & 0.580 \\
\hline$C_{30}(\mathrm{kPa})$ & 0.325 & -0.326 & -1.808 & 0.666 & -1.909 & 3.706 \\
\hline \multicolumn{7}{|l|}{ Mooney-Rivlin } \\
\hline$C_{1}(\mathrm{kPa})$ & -0.616 & 4.07 & -0.827 & 0.746 & 11.71 & 2.206 \\
\hline$C_{2}(\mathrm{kPa})$ & 1.691 & -2.946 & 1.711 & 3.579 & -7.307 & 2.167 \\
\hline \multicolumn{7}{|l|}{ Veronda-Westmann } \\
\hline$A_{1} \alpha(\mathrm{kPa})$ & 0.880 & 1.980 & & 4.945 & 6.965 & 6.158 \\
\hline$B_{1} \beta(\mathrm{kPa})$ & 0.195 & -0.856 & 1.239 & -0.6203 & -2.563 & -1.933 \\
\hline$\alpha$ & 1.240 & 1.263 & -3.303 & 0.735 & 0.884 & 0.973 \\
\hline Vito & & & $\gamma$ & & & \\
\hline$A_{1}(\mathrm{kPa})$ & 0.772 & & 1.066 & 6.897 & 7.799 & 7.095 \\
\hline$\alpha$ & 1.183 & 1.200 & -0.075 & 0.720 & 0.876 & 0.866 \\
\hline$\beta$ & 0.209 & -0.520 & 0.904 & -0.092 & -0.311 & -0.271 \\
\hline \multicolumn{7}{|l|}{ Full 2nd Order Poly } \\
\hline$C_{10}(\mathrm{kPa})$ & & 2.322 & 0.872 & 4.302 & 8.414 & 5.138 \\
\hline$C_{01}(\mathrm{kPa})$ & .169 & -1.198 & 0.012 & 0.23 & -4.011 & -0.913 \\
\hline$C_{20}(\mathrm{kPa})$ & oo & 20.913 & 2.886 & -22.663 & 43.064 & -64.389 \\
\hline$C_{02}(\mathrm{kPa})$ & 3.505 & -1.543 & -1.777 & -11.200 & -4.860 & -40.227 \\
\hline$C_{11}(\mathrm{kPa})$ & -8.8778 & -21.068 & 1.088 & 33.163 & -42.487 & 103.813 \\
\hline Rivlin & & & & & & \\
\hline$C_{10}(\mathrm{kPa})$ & 1.698 & 1.428 & 0.312 & 6.362 & 6.185 & 6.724 \\
\hline$C_{01}(\mathrm{kPa})$ & -0.623 & -0.304 & 0.572 & -2.037 & -1.783 & -2.499 \\
\hline$C_{20}(\mathrm{kPa})$ & 1.242 & 2.042 & 1.067 & 3.031 & 4.263 & 3.805 \\
\hline
\end{tabular}




\section{To be submitted to Physics in Medicine and Biology}

the AE-SWE data in all three imaging planes. Now referring to the second expectation, the selection of Veronda-Westman model for AE-SWE could be justifiable because its parameters can be explained in terms of the small strain modulus and a parameter related to the representation of strain-stiffening behavior Samani and Plewes (2004), Goenezen et al. (2012).

We also found material parameters estimated from AE-SWE and uniaxial compression data could be significantly different though the same material model was used (see Table 2). For instance, when the classic Mooney-Rivlin model was used, in the tissuemimicking gelatin, estimated $C_{1}$ and $C_{2}$ values were inconsistent, whereas parameters estimated from the Arruda-Boyce model were remarkably consistent. This is not entirely surprising because the SWS data acquired from three image planes and uniaxial compression data were used to estimate hyperelastic parameters, respectively. In the continuum mechanics literature, it is well understood that estimated hyperelastic parameters in some SEFs could be sensitive to the loading conditions (Ogden et al., 2004). Because there are great interests in estimating those nonlinear hyperelastic parameters using both nonlinear modulus inversion (Goenezen et al., 2012, Samani and Plewes, 2004) and AE-SWE data (Jiang et al., 2015a, Aristizabal et al., 2018), evaluating the consistency between two different approaches, i.e. mechanical testing, and AE-SWE data may shed light on this topic. In the future, this topic should be expanded into a comparison between nonlinear modulus inversion and hyperelastic parameter estimation using AE-SWE data.

In this study, SEFs were evaluated relative to their ability to model all three wave orienations represented in Fig. 2 simultaneously. Given the availability of 3D ultrasound data, it is feasible to obtain 3D elastographic data in the clinical workflow (Wang et al., 2013, Peng et al., 2017, Gennisson et al., 2015), though this capability is still emerging. Since SWE data in the clinical workflow typically were acquired from one of those three image planes, we also investigated this matter and found that, using only AE-SWE data acquired from one plane, the estimated nonlinear elastic parameters could substantially vary compared to those obtained by using 3 planes of AE-SWE Data (Table 2 vs. Table 4). By observation, we noted that, for most of the models, fitting to $c_{21}$ alone seemed to produce parameter estimates in better agreement with the uniaxial results than the other two orientations. This would suggest that while parameter estimation from $c_{21}$ alone may not capture the full constitutive characteristics relevant to AE-SWE, it may still produce a useful characterization of the uniaxial behavior of the material. This is important since $c_{21}$ is most commonly used for AE-SWE characterization.

Furthermore, it is probably wise to be cautious when AE-SWE data are acquired from different scanning views relative to a known or suspected load, since SWS will depend on the propagation direction relative to the load (e.g. $c_{12}$ versus $c_{23}$ ). For instance, liver ultrasound scanning can be done from multiple views (e.g. parasagittal scan ys. subcoastal scan) and a variety of factors known to alter SWS, such as phase of the subject's breath cycle and posture (Goertz et al., 2012), could be related to deformation of the liver. Acoustoelastic theory would suggest that variation due to 


\section{To be submitted to Physics in Medicine and Biology}

these factors should also depend on the ultrasonic scanning plane (i.e. plane of shear wave propogation) used at the time of measurement.

Equations used here are adopted from Ogden (Ogden, 2007) and are appropriate for a simplified scenario, i.e. a plane shear wave propagation in $2 \mathrm{D}$ isotropic, incompressible and homogeneous media. Some issues have to be addressed if we want to apply this theory to biological tissues. First, complicated wave propagation phenomena such as mode conversion and wave splitting existing in heterogeneous and perhaps locally anisotropic media have not been counted. Second, only shear wave propagation in planes of axial deformation (i.e. stretches along constant and spatially uniform principle axis) were considered. Given lessons learned from the rubber mechanics literature, further evaluations of those SEFs under more complex loading conditions (e.g. the inclusion of shearing) are necessary. Based on several excellent reviews (e.g. (Boyce and Arruda, 2000, Martins et al., 2006, Steinmann et al., 2012)), it was reported that "a large number of well-known SEFs are not reliable on the entire ranges of strain and different modes of deformation, simultaneously" (Mansouri and Darijani, 2014). Particularly, significant errors between SEF-predictions and mechanical testing data were observed when silicone rubber and soft tissues were deformed between $0-25 \%$ uniaxial deformation (Martins et al., 2006). Further errors could be introduced given more complex loading conditions. For instance, local shearing is common when heterogeneous tissues are deformed.

Given our preliminary data reported above, further studies will be focused on two aspects using biological tissues. First, further evaluations of SEFs under more complex loading/stress conditions will be performed. Second, comparing hyperelastic parameters estimated by the AE-SWE method and the uniaxial compression test method (Samani and Plewes, 2004) will also be conducted.

\section{Conclusion}

It was observed that, once all three image planes are considered, SEFs including the second invariant model tissue-mimicking materials better. Also, regardless of material models, material parameters calculated based on uni-axial compression could be quite different as compared to material parameters inversely estimated from AE-SWE data when the same material model was used. Implications of this observation should be further examined for inversely estimating nonlinear elastic parameters in strain elastography and SWE.

\section{Acknowledgment}

This project is partially funded by research grants from the US National Institutes of Health (R15CA179409 and R15EB026197). David Rosen was supported in part by a Portage Health Foundation Research Fellowship in Fall 2018, and is currently funded by 
To be submitted to Physics in Medicine and Biology

a Finishing Fellowship from the Michigan Technological University. The authors would like to thank undergraduate assistants (particularly, Derrick Diver and Lydia Kugler) for their assistance with phantom construction and measurement.

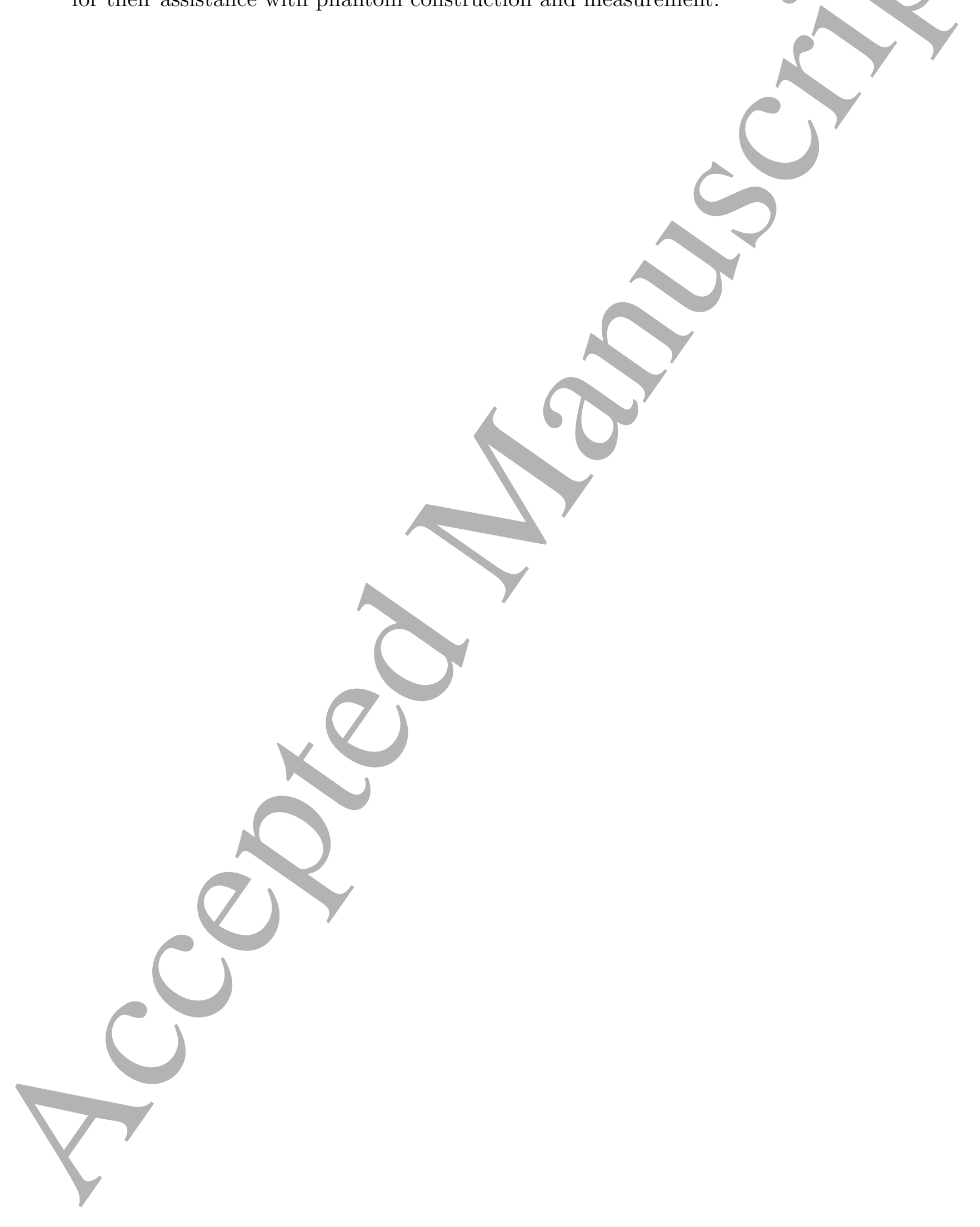




\section{References}

Aristizabal, S., Carrascal, C. A., Nenadic, I. Z., Greenleaf, J. F. and Urban, M. W. (2018). Application of acoustoelasticity to evaluate nonlinear modulus in ex vivo kidneys, IEEE Transactions on Ultrasonics, Ferroelectrics, and Frequency Control. 65(2): 188-200.

Arruda, E. M. and Boyce, M. C. (1993). A three-dimensional constitutive model for the large stretch behavior of rubber elastic materials, Journal of the Mechanics and Physics of Solids 41(2): 389-412.

Barr, R. G., Ferraioli, G., Palmeri, M. L., Goodman, Z. D., Garcia-Tsao, G., Rubin, J., Garra, B., Myers, R. P., Wilson, S. R., Rubens, D. and Levine, D. (2015). Elastography assessment of liver fibrosis: Society of radiologists in ultrasound consensus conference statement, Radiology 276(3): 845-861. PMID: 26079489.

Bercoff, J., Tanter, M. and Fink, M. (2004). Supersonic shear imaging: a new technique for soft tissue elasticity mapping, IEEE Transactions on Ultrasonics, Ferroelectrics, and Frequency Control 51(4): 396-409.

Bernal, M., Chamming's, F., Couade, M., Bercoff, J., Tanter, M. and Gennisson, J. (2016). In vivo quantification of the nonlinear/shear modulus in breast lesions: Feasibility study, IEEE Transactions on Ultraşonics, Ferroelectrics, and Frequency Control 63(1): 101-109.

Boyce, M. C. and Arruda, E. M. (2000). Constitutive models of rubber elasticity: A review, Rubber Chemistry and Technology 73(3): 504-523.

Carlson, L. C., Romero, S. T., Palmeri, M. L., Muñoz del Rio, A., Esplin, S. M., Rotemberg, V. M., Hall, T. J. and Feltovich, H. (2015). Changes in shear wave speed pre- and post-induction of labor: a feasibility study, Ultrasound in Obstetrics and Gynecology 46(1): 93-98.

Chang, J. M., Won, J.-K., Lee, K.-B., Park, I. A., Yi, A. and Moon, W. K. (2013). Comparison of shear-wave and strain ultrasound elastography in the differentiation of benign and malignant breast lesions, American Journal of Roentgenology 201(2): W347-W356.

Demiray, H. (1972). A note on the elasticity of soft biological tissues, J Biomech 5: 2.

DeWall, R.J., Jiang, J., Wilson, J. J. and Lee, K. S. (2014). Visualizing tendon elasticity in an ex vivo partial tear model, Ultrasound in Medicine and Biology 40(1): 158 167.

Fung, Y. Q. (1967). Elasticity of soft tissues in simple elongation, American Journal of Physiology-Legacy Content 213(6): 1532-1544.

Gennisson, J.-L., Deffieux, T., Fink, M. and Tanter, M. (2013). Ultrasound elastography: Principles and techniques, Diagnostic and Interventional Imaging 94(5): 487 - 495. Ultrasound elastography. 


\section{REFERENCES}

Gennisson, J. L., Renier, M., Catheline, S., Barriere, C., Bercoff, J., Tanter, M. and Fink, M. (2007). Acoustoelasticity in soft solids: Assessment of the nonlinear shear modulus with the acoustic radiation force, Journal of the Acoustical Society of America 122(6): 3211-3219.

Gennisson, J., Provost, J., Deffieux, T., Papadacci, C., Imbault, M., Pernot, M. and Tanter, M. (2015). 4-D ultrafast shear-wave imaging, IEEE Transactions on Ultrasonics, Ferroelectrics, and Frequency Control 62(6): 1059-1065.

Goenezen, S., Dord, J., Sink, Z., Barbone, P. E., Jiang, J., Hall, T. J. and Oberai, A. A. (2012). Linear and nonlinear elastic modulus imaging: An application to breast cancer diagnosis, IEEE Transactions on Medical Imaging 31(8): 1628-1637.

Goertz, R. S., Egger, C., Neurath, M. F. and Strobel, D. (2012). Impact of food intake, ultrasound transducer, breathing maneuvers and body position on acoustic radiation force impulse (arfi) elastometry of the liver, Ultraschall in Med 33(04): 380-385.

Hall, T. J., Bilgen, M., Insana, M. F. and Krouskop, T. A. (1997). Phantom materials for elastography, IEEE Transactions on Ultrasonics, Ferroelectrics, and Frequency Control 44(6): 1355-1365.

Hug, F., Tucker, K., Gennisson, J.-L., Tanter, M. and Nordez, A. (2015). Elastography for Muscle Biomechanics: Toward the Estimation of Individual Muscle Force, Exercise and Sport Science Reviews 43(3): 125-133.

Jiang, Y., Li, G., Qian, L.-X., Liang, S., Destrade, M. and Cao, Y. (2015a). Measuring the linear and nonlinear elastic properties of brain tissue with shear waves and inverse analysis, Biomechanics and Modeling in Mechanobiology 14(5): 1119-1128.

Jiang, Y., Li, G.-Y., Qian, L.-X., Hu, X.-D., Liu, D., Liang, S. and Cao, Y. (2015b). Characterization of the nonlinear elastic properties of soft tissues using the supersonic shear imaging (ssi) technique: Inverse method, ex vivo and in vivo experiments, Medical Image Analysis 20(1): 97-111.

Joseph, J. O. and Abbas, S. (2009). Measurement of the hyperelastic properties of 44 pathological ex vivo breast tissue samples, Physics in Medicine E Biology 54(8): 2557.

Mansouri, M. and Darijani, H. (2014). Constitutive modeling of isotropic hyperelastic materials in an exponential framework using a self-contained approach, International Journal of Solids and Structures 51(25): 4316 - 4326.

Martins, P.A. L. S., Natal Jorge, R. M. and Ferreira, A. J. M. (2006). A comparative study of several material models for prediction of hyperelastic properties: Application to silicone-rubber and soft tissues, Strain 42(3): 135-147.

Mooney, M. (1940). A theory of large elastic deformation, Journal of Applied Physics 11(9): 582-592.

Nightingale, K., McAleavey, S. and Trahey, G. (2003). Shear-wave generation using acoustic radiation force: in vivo and ex vivo results, Ultrasound in Medicine and Biology 29(12): 1715 - 1723 . 


\section{REFERENCES}

Norris, A. (2007). Small-on-Large Theory with Applications to Granular Materials and Fluid/Solid Systems, Springer Vienna, Vienna, pp. 27-62.

Ogden, R. W. (2007). Incremental statics and dynamics of pre-stressed elastic materials., pp. $1-27$.

Ogden, R. W. (2013). Non-Linear Elastic Deformations, Courier Corporation.

Ogden, R. W., Saccomandi, G. and Sgura, I. (2004). Fitting hyperelastic models to experimental data, Computational Mechanics 34(6): 484-502.

Peng, B., Wang, Y., Hall, T. J. and Jiang, J. (2017). A gpu-accelerated 3-d coupled subsample estimation algorithm for volumetric breast strain elastography, IEEE Transactions on Ultrasonics, Ferroelectrics, and Frequency Control 64(4): 694-705.

Rivlin, R. S. (1948). Large elastic deformations of isotropic materials. iv. further developments of the general theory, Philosophical Transactions of the Royal Society of London. Series A, Mathematical and Physical Sciences 241(835): 379-397.

Rivlin, R. S. and Saunders, D. W. (1951). Large elastic deformations of isotropic materials vii. experiments on the deformation of rubber., Philosophical Transactions of the Royal Society of London. Series A, Mathematical and Physical Sciences 243(865): 251-288.

Rosen, D. and Jiang, J. (2018). Fourier-domain shift matching: A robust time-offlight approach for shear wave speed estimation, IEEE Transactions on Ultrasonics, Ferroelectrics, and Frequency Control 65(5): 729-740.

Samani, A. and Plewes, D. (2004). A method to measure the hyperelastic parameters of ex vivo breast tissue samples, Physics in Medicine and Biology 49(18): 4395.

Sarvazyan, A. P., Rudenko, O. V., Swanson, S. D., Fowlkes, J. and Emelianov, S. Y. (1998). Shear wave elasticity imaging: a new ultrasonic technology of medical diagnostics, Ultrasound in Medicine and Biology 24(9): 1419 - 1435.

Shiina, T., Nightingale, K. R., Palmeri, M. L., Hall, T. J., Bamber, J. C., Barr, R. G., Castera, L., Choi, B. I., Chou, Y.-H., Cosgrove, D., Dietrich, C. F., Ding, H., Amy, D., Farrokh, A., Ferraioli, G., Filice, C., Friedrich-Rust, M., Nakashima, K., Schafer, F., Sporea, I., Suzuki, S., Wilson, S. and Kudo, M. (2015). \{WFUMB $\}$ guidelines and recommendations for clinical use of ultrasound elastography: Part 1: Basic principles and terminology, Ultras,und in Medicine Ef Biology 41(5): 1126 - 1147.

Steinmann, P., Hossain, M. and Possart, G. (2012). Hyperelastic models for rubber-like materials: consistent tangent operators and suitability for treloar's data, Archive of Applied Mechanics 82(9): 1183-1217.

Urban, M. W., Lopera, M., Aristizabal, S., Amador, C., Nenadic, I., Kinnick, R. R., Weston, A. D., Qiang, B., Zhang, X. and Greenleaf, J. F. (2014). Characterization of transverse isotropy in compressed tissue mimicking phantoms, IEEE transactions on ultrasonics, ferroelectrics, and frequency control 2014: 1834-1837.

Veronda, D. R. and Westmann, R. A. (1970). Mechanical characterization of skin-finite deformations, J Biomech 3(1): 111-24. 


\section{REFERENCES}

Vito, R. (1973). A note on arterial elasticity, J Biomech 6(5): 561-4.

Wang, M., Byram, B., Palmeri, M., Rouze, N. and Nightingale, K. (2013). Imaging transverse isotropic properties of muscle by monitoring acoustic radiation force induced shear waves using a 2-d matrix ultrasound array, IEEE Transactions, on Medical Imaging 32(9): 1671-1684.

Wex, C., Arndt, S., Stoll, A., Bruns, C. and Kupriyanova, Y. (2015). Isotropic incompressible hyperelastic models for modelling the mechanical behaviour of biological tissues: a review, Biomedical Engineering-Biomedizinische Technik 60(6): $577-592$.

Yeoh, O. H. (1990). Characterization of elastic properties of carbon-black-filled rubber vulcanizates, Rubber Chemistry and Technology 63(5): 792-805. 